\title{
Information demand and cryptocurrency market activity
}

Article

Accepted Version

Creative Commons: Attribution-Noncommercial-No Derivative Works 4.0

Katsiampa, P., Moutsianas, K. and Urquhart, A. (2019) Information demand and cryptocurrency market activity. Economics Letters, 185. 108714. ISSN 0165-1765 doi: https://doi.org/10.1016/j.econlet.2019.108714 Available at https://centaur.reading.ac.uk/86347/

It is advisable to refer to the publisher's version if you intend to cite from the work. See Guidance on citing.

To link to this article DOI: http://dx.doi.org/10.1016/j.econlet.2019.108714

Publisher: Elsevier

All outputs in CentAUR are protected by Intellectual Property Rights law, including copyright law. Copyright and IPR is retained by the creators or other copyright holders. Terms and conditions for use of this material are defined in the End User Agreement.

\section{www.reading.ac.uk/centaur}

\section{CentAUR}

Central Archive at the University of Reading

Reading's research outputs online 


\title{
Information demand and cryptocurrency market activity
}

\begin{abstract}
:
This paper studies the relationship between information demand measured by Google search volume index, price returns, and trading volume for five major cryptocurrencies. We find that past information demand flows significantly influence the volume of all cryptocurrencies except for Litecoin. Moreover, trading volumes are found to Granger cause the information demand flows of Bitcoin, Ripple, and Litecoin, while previous day's returns significantly influence the information demand flows of all the altcoins.
\end{abstract}

Keywords: Information demand flows, Bitcoin, Cryptocurrency, Volume, VAR

\section{Introduction}

The importance of information to market activity has been highlighted in several studies. Information is the most valued asset in financial markets (Vlastakis and Markellos, 2012). For this reason, there has been increased academic interest in the relationship between information demand and market activity in different financial markets (see, e.g., Vlastakis and Markellos, 2012; Vozlyublennaia, 2014; Goddard et al., 2015; Chronopoulos et al., 2018). Nevertheless, the relationship between information demand and market activity in cryptocurrency markets still remains underexplored, despite the fact that the literature on cryptocurrencies has rapidly emerged. Among the few studies that have analysed cryptocurrency search queries are those of Ciaian et al. (2016), Urquhart (2018), and Shen et al. (2019). However, all of these studies considered only Bitcoin excluding other cryptocurrencies despite the fact that altcoins have been gaining in popularity and market share. ${ }^{1}$

Consequently, this paper studies the relationship between information demand, returns, and trading volume for five major cryptocurrencies. Our study contributes to the growing literature on cryptocurrencies as well as on the relationship between information demand and market activity. To the best of the authors' knowledge, this is the first study to examine such a relationship for altcoins.

\footnotetext{
${ }^{1}$ It is worth mentioning that while Bitcoin's market share was about $95 \%$ in May 2013, it has significantly dropped to $55 \%$ (Coinmarketcap.com accessed on $12^{\text {th }}$ June 2019).
} 


\section{Data and methodology}

In this study, we consider the five largest cryptocurrencies in terms of market capitalisation $^{2}$. Data on cryptocurrencies' price and volume are collected from the earliest date for which both price and volume data are available at https://coinmarketcap.com/coins/ to $20^{\text {th }}$ January 2019. The dataset therefore comprises daily closing prices and volumes for Bitcoin (from $27^{\text {th }}$ December 2013), Ripple (from $27^{\text {th }}$ December 2013), Ether (from $7^{\text {th }}$ August 2015), Stellar (from $5^{\text {th }}$ August 2014), and Litecoin (from $27^{\text {th }}$ December 2013). Similar to Urquhart (2018), daily data about information demand flows are sourced from Google Trends using the name of the cryptocurrency as the keyword.

In order to explore the dynamics between our variables, we employ the Vector Autoregressive (VAR) model, expressed as

$$
y_{t}=c+\sum_{i=1}^{p} \beta_{i} y_{t-i}+\varepsilon_{t}
$$

where $y_{t}$ is a $3 \times 1$ vector containing the three variables of our interest (i.e., returns ${ }^{3}$, logarithmic volumes, and standardised information demand flows ${ }^{4}$ ), $c$ denotes a $3 \times 1$ vector of constants, and $\varepsilon_{t}$ represents a $3 \times 1$ vector of independent white noise errors. The lag order $p$ is determined using the Schwarz information criterion allowing up to ten lags. In order to investigate possible causal relationships between information demand, volume, and returns, we also employ Granger causality tests.

\section{Empirical results}

\subsection{Data analysis}

Summary statistics for our variables can be found in Table 1. We notice that all the cryptocurrencies have a positive mean return, while Bitcoin has the smallest standard deviation and Stellar the highest. ${ }^{5}$

\footnotetext{
${ }^{2}$ As of $21^{\text {st }}$ January 2019.

${ }^{3}$ As standard, we compute logarithmic returns.

${ }^{4}$ Standardised as (value - mean)/standard deviation.

${ }^{5}$ In unreported results due to space constraints, we ensure that our variables are stationary through the Phillips-Perron test. These results are available upon request from the corresponding author.
} 


\subsection{Entire sample period analysis}

In Table 3, Panel A presents the estimation results of the VAR models for each cryptocurrency over the entire sample period, while Panel B provides the corresponding Granger causality test statistics. Unlike the volume and information demand equations, in the return equation we find little evidence of autocorrelation of returns. With the exception of Ether, we further find little evidence that previous day's volume or information demand predicts returns, where these findings are supported by the Granger causality results in Panel B. For the volume equation, we find significant evidence that previous day's return predicts volume for all altcoins, while previous day's information demand predicts volume for Ether and Stellar. The information demand equation shows that, except for Bitcoin, previous day's return predicts the following day's demand. Also, previous day's volume predicts information demand for all cryptocurrencies except Stellar.

\subsection{Sub-sample analysis}

Next, we performed the multiple unknown structural breakpoint test of Bai and Perron (1998) and found that Bitcoin and Ether exhibit one breakpoint each on $17^{\text {th }}$ December 2017 and $14^{\text {th }}$ January 2018, respectively. Consequently, we re-performed our analysis in the sub-samples for Bitcoin and Ether, the results of which can be found in Table 3. Overall, we notice some discrepancies when comparing the two sub-samples not only in the magnitude, sign, and significance of the different coefficients (Panel A) but also in the Granger causality results (Panel B).

Regarding Bitcoin, similar to the entire sample, information demand does not have a significant effect in the returns in any sub-sample. Nevertheless, previous day's returns are now found to have a significant impact on Bitcoin's information demand in both sub-samples, although in the first sub-sample this is true only at the $10 \%$ level of significance. Furthermore, similar to the entire sample, past volume significantly influences current information demand in both sub-samples, whereas previous information demand flows now have a significant impact on current trading volume levels only in the first sub-sample. All these findings are in agreement with the Granger causality results. The Granger causality results further reveal significant bidirectional causality between returns and volume but only in the first sub-sample. 
As for Ether, in the return equation we find a significant estimate of the autoregressive parameter for information demand for lag 1 at the $5 \%$ level in the sub-sample before the breakpoint but not in the second sub-sample. It is worth mentioning that information demand flows were found to Granger-cause returns in the entire sample period as well. Moreover, similar to the entire period, past returns significantly influence the current information demand in both sub-sample periods, as well as the current trading volume but only in the first sub-sample. We also notice that, similar to the entire period, past information demand significantly affects current trading volume in both sub-periods. All these findings are in accordance with the Granger causality results. On the other hand, similar to the entire sample, volume does not Granger-cause information demand in any sub-sample. However, unlike the results for the entire sample period, the Granger causality results for the sub-samples suggest that volume does not Granger cause the returns in any of the two sub-samples.

\section{Conclusion}

This paper examined the relationship between information demand, returns, and trading volumes for five cryptocurrencies by employing the VAR model and the Granger causality test. According to the results over the entire sample period, previous day's returns significantly influence the information demand flows of all cryptocurrencies apart from Bitcoin and previous day's volume significantly affects the information demand of all cryptocurrencies except for Stellar. On the other hand, information demand flows at lags 1 or 2 significantly influence the volume of all cryptocurrencies except for Litecoin but the returns of only Ether.

However, after testing for structural breaks and separating the sample period of Bitcoin and Ether in two, we found that returns influence information demand in both subsamples of both cryptocurrencies, with the causality being stronger in the sub-sample after the breakpoint, though. Moreover, volume Granger-causes information demand in both sub-samples of Bitcoin but not in any of the two sub-samples of Ether. Finally, it was shown that past information demand flows influence the returns of Ether in the first sub-sample as well as the volume of Ether in both sub-samples and the volume of Bitcoin in the first sub-sample. 


\section{References}

Bai, J.,\& Perron, P. (1998). Estimating and testing linear models with multiple structural changes. Econometrica, 47-78.

Ciaian, P., Rajcaniova, M.,\& Kancs, D.A. (2016). The economics of BitCoin price formation. Applied Economics, 48(19), 1799-1815.

Chronopoulos, D.K., Papadimitriou, F.I.,\& Vlastakis, N. (2018). Information demand and stock return predictability. Journal of International Money and Finance, 80, 5974.

Goddard, J., Kita, A.,\& Wang, Q. (2015). Investor attention and FX market volatility. Journal of International Financial Markets, Institutions and Money, 38, 79-96.

Shen, D., Urquhart, A.,\& Wang, P. (2019). Does twitter predict Bitcoin?. Economics Letters, 174, 118-122.

Vlastakis, N.,\& Markellos, R.N. (2012). Information demand and stock market volatility. Journal of Banking \& Finance, 36(6), 1808-1821.

Vozlyublennaia, N. (2014). Investor attention, index performance, and return predictability. Journal of Banking \& Finance, 41, 17-35.

Urquhart, A. (2018). What causes the attention of Bitcoin?. Economics Letters, 166, 40-44. 
Table 1 Summary statistics.

\begin{tabular}{cccccc}
\hline & Bitcoin & Ripple & Ether & Stellar & Litecoin \\
\hline Return & & & & \\
\hline Mean & 0.0009 & 0.0013 & 0.0030 & 0.0023 & 0.0002 \\
St.Dev. & 0.0395 & 0.0704 & 0.0778 & 0.0807 & 0.0590 \\
Max & 0.2251 & 1.0274 & 0.4123 & 0.7231 & 0.5103 \\
Min & -0.2376 & -0.6163 & -1.3021 & -0.3664 & -0.5139 \\
Skewness & -0.4194 & 2.3549 & -3.3796 & 1.9354 & 0.5365 \\
Kurtosis & 8.5386 & 39.9188 & 67.5033 & 17.9068 & 15.8817 \\
\hline Log-Volume & & & & \\
\hline Mean & 19.0892 & 15.4149 & 18.4436 & 13.2183 & 16.5895 \\
St.Dev. & 2.3170 & 3.2726 & 2.9631 & 3.7403 & 2.4052 \\
Max & 23.8947 & 22.9327 & 22.9441 & 21.1375 & 22.6637 \\
Min & 14.8656 & 9.0259 & 11.5340 & 6.1964 & 13.0851 \\
Skewness & 0.4715 & 0.5084 & -0.4277 & 0.3023 & 0.4995 \\
Kurtosis & 1.7102 & 1.7612 & 1.9019 & 1.5612 & 1.6308 \\
\hline Stan-Idf & & & & \\
\hline Mean & 0.0000 & 0.0000 & 0.0000 & -0.0000 & -0.0000 \\
St.Dev. & 1.0003 & 1.0003 & 1.0004 & 1.0003 & 1.0003 \\
Max & 3.6064 & 1.8696 & 3.1577 & 2.4826 & 3.1567 \\
Min & -1.6420 & -2.1239 & -1.5358 & -1.7908 & -1.4135 \\
Skewness & 0.5268 & -0.5980 & 0.7027 & 0.1916 & 0.6006 \\
Kurtosis & 3.2327 & 2.1621 & 3.0521 & 2.0923 & 2.7624 \\
\hline
\end{tabular}


Table 2 Results for the entire sample.

\begin{tabular}{|c|c|c|c|c|c|}
\hline & Bitcoin & Ripple & Ether & Stellar & Litecoin \\
\hline \multicolumn{6}{|c|}{ Panel A: Estimation results } \\
\hline \multicolumn{6}{|l|}{ Return } \\
\hline$R_{t-1}$ & 0.0001 & 0.0097 & 0.0432 & $0.0537 * *$ & -0.0075 \\
\hline$R_{t-2}$ & -0.0368 & $0.0630 * * *$ & 0.0225 & -0.0370 & $-0.0525 * *$ \\
\hline$R_{t-3}$ & 0.0358 & 0.0368 & -0.028 & 0.0343 & 0.0322 \\
\hline$R_{t-4}$ & -0.0179 & 0.0151 & & -0.0278 & \\
\hline$R_{t-5}$ & 0.0134 & $0.0422 *$ & & $0.0631 * *$ & \\
\hline$R_{t-6}$ & $0.0531 * *$ & $0.0463 * *$ & & 0.0208 & \\
\hline$R_{t-7}$ & & & & 0.0007 & \\
\hline$V o l_{t-1}$ & 0.0031 & 0.0016 & $0.0091 * *$ & $0.0059 *$ & 0.0013 \\
\hline$V_{o l} l_{t-2}$ & -0.0014 & 0.0026 & 0.0017 & -0.0009 & -0.0003 \\
\hline$V_{o l} l_{t-3}$ & 0.0018 & $-0.0074 * *$ & $-0.0115 * *$ & $-0.0069 *$ & -0.0011 \\
\hline$V o l_{t-4}$ & 0.0015 & 0.0001 & & 0.0039 & \\
\hline$V o l_{t-5}$ & -0.0009 & -0.0013 & & -0.0004 & \\
\hline$V_{o l} l_{t-6}$ & -0.0040 & 0.0038 & & -0.0058 & \\
\hline Vol $_{t-7}$ & & & & 0.0039 & \\
\hline$I d f_{t-1}$ & -0.0005 & -0.0004 & $-0.0100 *$ & -0.0020 & -0.0012 \\
\hline$I d f_{t-2}$ & -0.0032 & 0.0014 & 0.0027 & -0.0031 & 0.0013 \\
\hline$I d f_{t-3}$ & 0.0034 & -0.0040 & 0.0022 & 0.0075 & -0.0038 \\
\hline$I d f_{t-4}$ & 0.0039 & -0.0016 & & 0.0021 & \\
\hline$I d f_{t-5}$ & $-0.0065 * *$ & -0.0056 & & -0.0077 & \\
\hline$I d f_{t-6}$ & 0.0026 & 0.0045 & & 0.0005 & \\
\hline$I d f_{t-7}$ & & & & -0.0019 & \\
\hline Constant & -0.0002 & 0.0116 & 0.0165 & 0.0063 & 0.0014 \\
\hline \multicolumn{6}{|l|}{ Volume } \\
\hline$R_{t-1}$ & 0.0707 & $0.6901 * * *$ & $0.6200 * * *$ & $1.7310 * * *$ & $0.3479 *$ \\
\hline$R_{t-2}$ & $0.5316^{* *}$ & $0.3860 * *$ & 0.0028 & $0.3783^{*}$ & $0.3145^{*}$ \\
\hline$R_{t-3}$ & 0.3146 & $0.4794 * *$ & -0.1705 & 0.1956 & 0.0634 \\
\hline$R_{t-4}$ & 0.1557 & 0.1552 & & 0.1728 & \\
\hline$R_{t-5}$ & 0.2402 & 0.0322 & & 0.2038 & \\
\hline$R_{t-6}$ & 0.0363 & $-0.3417 *$ & & $-0.4946 * *$ & \\
\hline$R_{t-7}$ & & & & -0.2911 & \\
\hline $\operatorname{Vol}_{t-1}$ & $0.6190 * * *$ & $0.6910 * * *$ & $0.6273^{*} * *$ & $0.5567 * * *$ & $0.7524 * * *$ \\
\hline Vol $_{t-2}$ & 0.0200 & -0.0008 & $0.1416 * * *$ & $0.1118 * * *$ & 0.0072 \\
\hline $\operatorname{Vol}_{t-3}$ & $0.1071 * * *$ & $0.0487 *$ & $0.2244 * * *$ & $0.0978 * * *$ & $0.2262 * * *$ \\
\hline$V o l_{t-4}$ & $0.0785 * * *$ & $0.0518^{*}$ & & 0.0250 & \\
\hline$V o l_{t-5}$ & 0.0036 & 0.0255 & & $0.0845^{* * *}$ & \\
\hline
\end{tabular}




\begin{tabular}{|c|c|c|c|c|c|}
\hline $\operatorname{Vol}_{t-6}$ & $0.1666 * * *$ & $0.1669 * * *$ & & 0.0237 & \\
\hline$V_{o l} l_{t-7}$ & & & & $0.0906^{* * *}$ & \\
\hline$I d f_{t-1}$ & 0.0265 & 0.0592 & $0.1300 * * *$ & $0.1101 * * *$ & -0.0201 \\
\hline$I d f_{t-2}$ & $-0.0572 * *$ & $-0.1270 * * *$ & $-0.1243 * * *$ & $-0.1144 * *$ & -0.0022 \\
\hline$I d f_{t-3}$ & 0.0023 & -0.0469 & -0.0369 & -0.0117 & -0.0073 \\
\hline$I d f_{t-4}$ & -0.0069 & -0.0149 & & $-0.0938^{*}$ & \\
\hline$I d f_{t-5}$ & -0.0273 & $0.0785^{*}$ & & 0.0616 & \\
\hline$I d f_{t-6}$ & $0.0484 * *$ & 0.0004 & & -0.0101 & \\
\hline$I d f_{t-7}$ & & & & 0.0333 & \\
\hline Constant & 0.1064 & $0.2689 * * *$ & $0.1312 *$ & $0.1358 * *$ & $0.2381 * * *$ \\
\hline Idf & & & & & \\
\hline$R_{t-1}$ & -0.0710 & $0.4580 * * *$ & $0.4076^{* *}$ & $0.6870 * * *$ & $0.6147 * * *$ \\
\hline$R_{t-2}$ & $0.4207 *$ & 0.1514 & $0.3784 * *$ & $0.3686^{* * *}$ & 0.0217 \\
\hline$R_{t-3}$ & 0.0625 & $0.1871^{*}$ & $0.2708^{*}$ & 0.0939 & 0.1170 \\
\hline$R_{t-4}$ & $0.4180^{*}$ & 0.0532 & & -0.0508 & \\
\hline$R_{t-5}$ & -0.1841 & -0.1515 & & 0.1167 & \\
\hline$R_{t-6}$ & 0.2400 & -0.0443 & & -0.1072 & \\
\hline$R_{t-7}$ & & & & $-0.3242 * * *$ & \\
\hline$V_{o l} l_{t-1}$ & $0.0771 * * *$ & $0.0615 * * *$ & $0.0634 * *$ & -0.0259 & $0.0887 * * *$ \\
\hline$V_{o l} l_{t-2}$ & $-0.0536^{*}$ & $-0.0521 * * *$ & $-0.0554 *$ & -0.0168 & -0.0391 \\
\hline$V_{o l} l_{t-3}$ & 0.0341 & -0.0191 & -0.0048 & 0.0082 & $-0.0604 * *$ \\
\hline$V o l_{t-4}$ & $-0.0536^{*}$ & 0.0082 & & -0.0140 & \\
\hline$V o l_{t-5}$ & -0.0355 & $-0.0370 * *$ & & $0.0383 * *$ & \\
\hline$V o l_{t-6}$ & 0.0257 & $0.0317 * *$ & & 0.0035 & \\
\hline $\operatorname{Vol}_{t-7}$ & & & & 0.0017 & \\
\hline$I d f_{t-1}$ & $0.7800 * * *$ & $0.5705 * * *$ & $0.7416^{* * *}$ & $0.6004 * * *$ & $0.4959 * * *$ \\
\hline$I d f_{t-2}$ & $-0.0948 * * *$ & $0.0454^{*}$ & 0.0312 & -0.0409 & $0.2062 * * *$ \\
\hline$I d f_{t-3}$ & $0.0994 * * *$ & $0.0911 * * *$ & $0.1592 * * *$ & $0.0964 * * *$ & $0.2058 * * *$ \\
\hline$I d f_{t-4}$ & 0.0452 & 0.0423 & & $0.0513^{*}$ & \\
\hline$I d f_{t-5}$ & 0.0326 & $0.0874 * * *$ & & -0.0343 & \\
\hline$I d f_{t-6}$ & $0.0875 * * *$ & $0.1285^{* * *}$ & & $0.0911 * * *$ & \\
\hline$I d f_{t-7}$ & & & & $0.1951 * * *$ & \\
\hline Constant & 0.1111 & $0.1026^{* *}$ & -0.0665 & 0.0634 & $0.1805^{* *}$ \\
\hline \multicolumn{6}{|c|}{ Panel B: Granger causality tests } \\
\hline $\begin{array}{l}\text { Ret does not } \\
\text { Granger-Cause } \\
\text { Stan-Idf } \\
\end{array}$ & 7.7669 & $24.958 * * *$ & $15.458 * * *$ & $43.406^{* * *}$ & $9.4696 * *$ \\
\hline $\begin{array}{l}\text { Log-Vol does not } \\
\text { Granger-Cause } \\
\text { Stan-Idf }\end{array}$ & $14.968 * *$ & $37.558 * * *$ & 5.8008 & $13.706^{*}$ & $19.593 * * *$ \\
\hline $\begin{array}{l}\text { Stan-Idf does not } \\
\text { Granger-Cause Ret }\end{array}$ & 7.7685 & 8.778 & $9.0327 * *$ & 7.5071 & $6.8551 *$ \\
\hline $\begin{array}{l}\text { Stan-Idf does not } \\
\text { Granger-Cause log- } \\
\text { Vol }\end{array}$ & $13.26^{* *}$ & $23.647 * * *$ & $26.019 * * *$ & $19.058 * * *$ & $7.1143 *$ \\
\hline
\end{tabular}




\begin{tabular}{|c|c|c|c|c|c|}
\hline $\begin{array}{l}\text { Ret does not } \\
\text { Granger-Cause log- } \\
\text { Vol }\end{array}$ & $10.865^{*}$ & $27.854 * * *$ & $12.776 * * *$ & $76.928 * * *$ & $6.672 *$ \\
\hline $\begin{array}{l}\text { Log-Vol does not } \\
\text { Granger-Cause Ret }\end{array}$ & 4.4503 & 7.7086 & $8.3529 * *$ & 9.6139 & 0.2841 \\
\hline
\end{tabular}

Note: $* * *$ and $* * *$ denote significance at the $10,5 \%$ and $1 \%$ level, respectively. 
Table 3 Results for the sub-samples.

\begin{tabular}{|c|c|c|c|c|}
\hline & \multicolumn{2}{|c|}{ Bitcoin } & \multicolumn{2}{|c|}{ Ether } \\
\hline & Before break & After break & Before break & After break \\
\hline \multicolumn{5}{|c|}{ Panel A: Estimation results } \\
\hline \multicolumn{5}{|l|}{ Return } \\
\hline$R_{t-1}$ & 0.0040 & -0.0446 & $0.0994 * *$ & 0.0038 \\
\hline$R_{t-2}$ & $-0.1001 * * *$ & 0.0679 & -0.0035 & 0.0330 \\
\hline$R_{t-3}$ & 0.0227 & 0.0524 & & 0.0582 \\
\hline $\operatorname{Vol}_{t-1}$ & 0.0032 & -0.0129 & $0.0100 *$ & -0.0026 \\
\hline $\operatorname{Vol}_{t-2}$ & -0.0037 & 0.0238 & $-0.0104 *$ & 0.0088 \\
\hline$V o l_{t-3}$ & 0.0035 & -0.0208 & & -0.0108 \\
\hline$I d f_{t-1}$ & -0.0011 & 0.0041 & $-0.0181 * *$ & 0.0044 \\
\hline$I d f_{t-2}$ & -0.0021 & -0.0072 & 0.0110 & -0.0088 \\
\hline$I d f_{t-3}$ & 0.0030 & 0.0045 & & 0.0046 \\
\hline Constant & $-0.0519 * * *$ & 0.2190 & 0.0103 & $0.0986^{* *}$ \\
\hline \multicolumn{5}{|l|}{ Volume } \\
\hline$R_{t-1}$ & 0.0630 & -0.0343 & $0.8365 * * *$ & 0.1904 \\
\hline$R_{t-2}$ & $0.6825^{* *}$ & 0.3302 & -0.0708 & 0.0569 \\
\hline$R_{t-3}$ & 0.4218 & 0.2187 & & 0.1128 \\
\hline$V_{o l} l_{t-1}$ & $0.6962 * * *$ & $0.6755 * * *$ & $0.6535 * * *$ & $0.6907 * * *$ \\
\hline $\operatorname{Vol}_{t-2}$ & $0.0619^{*}$ & 0.0687 & $0.2979 * * *$ & 0.0596 \\
\hline$V o l_{t-3}$ & $0.2231 * * *$ & $0.1793 * * *$ & & $0.2259 * * *$ \\
\hline$I d f_{t-1}$ & 0.0125 & 0.0338 & $0.0928 *$ & $0.0998 * *$ \\
\hline$I d f_{t-2}$ & $-0.0870 * *$ & -0.0459 & $-0.1577 * * *$ & $-0.1298 * *$ \\
\hline$I d f_{t-3}$ & 0.0421 & 0.0125 & & 0.0323 \\
\hline Constant & $0.3476 * * *$ & $1.7184 * * *$ & $0.7627 * * *$ & $0.4976 * *$ \\
\hline \multicolumn{5}{|l|}{ Idf } \\
\hline$R_{t-1}$ & $0.5111^{*}$ & $-1.9620 * * *$ & $0.5812 * *$ & $0.5024 * *$ \\
\hline$R_{t-2}$ & $0.5061 *$ & 0.0664 & 0.0655 & $0.4636^{* *}$ \\
\hline$R_{t-3}$ & 0.1678 & 0.4180 & & 0.2880 \\
\hline $\operatorname{Vol}_{t-1}$ & $0.0685^{* *}$ & $0.3322 * *$ & $0.0636^{*}$ & -0.0341 \\
\hline $\operatorname{Vol}_{t-2}$ & $-0.0543 *$ & $-0.4162 * *$ & $-0.0733 * *$ & -0.0040 \\
\hline $\operatorname{Vol}_{t-3}$ & -0.0255 & 0.0463 & & 0.0586 \\
\hline$I d f_{t-1}$ & $0.8096^{* * *}$ & $0.7306 * * *$ & $0.6267 * * *$ & $0.9560 * * *$ \\
\hline$I d f_{t-2}$ & $-0.0639 *$ & -0.0213 & $0.2593 * * *$ & $-0.2679 * * *$ \\
\hline$I d f_{t-3}$ & $0.1836 * * *$ & $0.2262 * * *$ & & $0.2486 * * *$ \\
\hline Constant & $0.2099 *$ & 0.8184 & 0.1431 & $-0.4236^{*}$ \\
\hline \multicolumn{5}{|c|}{ Panel B: Granger causality tests } \\
\hline $\begin{array}{l}\text { Ret does not } \\
\text { Granger-Cause } \\
\text { Stan-Idf }\end{array}$ & $6.9176^{*}$ & $15.054 * * *$ & $4.8743^{*}$ & $13.607 * * *$ \\
\hline
\end{tabular}




\begin{tabular}{lcccc}
\hline $\begin{array}{l}\text { Log-Vol does not } \\
\text { Granger-Cause } \\
\text { Stan-Idf }\end{array}$ & $10.375 * *$ & $7.9483 * *$ & 4.3067 & 4.9688 \\
\hline $\begin{array}{l}\text { Stan-Idf does not } \\
\text { Granger-Cause Ret }\end{array}$ & 1.5677 & 1.1404 & $8.0893 * *$ & 0.7688 \\
\hline $\begin{array}{l}\text { Stan-Idf does not } \\
\text { Granger-Cause log- } \\
\text { Vol }\end{array}$ & $14.189 * * *$ & 2.2045 & $11.522 * * *$ & $7.3429 *$ \\
\hline $\begin{array}{l}\text { Ret does not } \\
\text { Granger-Cause log- } \\
\text { Vol }\end{array}$ & $8.2154 * *$ & 3.1148 & $6.7432 * *$ & 1.4092 \\
\hline $\begin{array}{l}\text { Log-Vol does not } \\
\text { Granger-Cause Ret }\end{array}$ & $25.408 * * *$ & 4.7677 & 3.1364 & 6.1246 \\
\hline
\end{tabular}

Note: $* * *$ and $* * *$ denote significance at the $10,5 \%$ and $1 \%$ level, respectively. 